\title{
Diagnostic value of Hyaluronic acid in patients with chronic obstructive pulmonary diseases
}

\author{
A.A.Okab, M.H.Kame, A.M.Abdelrahman, M.E.El nagger and D.A.Abdel Hafez \\ Chest, Dept., Faculty of Medicine, Banha Univ., Egypt \\ E-Mail:doaa153@gmail.com
}

\begin{abstract}
Background: COPD is an important and increasing cause of mortality, it was estimated to be the fifth leading cause of death. The aim of this work is to study the value of hyaluronic acid (HA) in patients with chronic obstructive pulmonary disease (COPD) and follow up its level with medications in these patients. Patients and methods: This cross-sectional study was carried out in chest department at Benha university hospitals, the samples were collected from patients in the period from Mars 2019 to December 2020. All subjects underwent history talking and clinical examination, plain chest x-ray (postero-anterior) and (left-lateral) view and PFT (spirometery) before and after bronchodilator and it will be repeated at 3 and 6 months with the assay HA Results: there was statistically significant decrease in Hyaluronic acid in visit two than visit one, and in visit three than visit one and two, and there was statistically significant difference between the controls and the two sub groups as regards Hyaluronic acid, where control is the lowest then subgroup 1 then subgroup 2 in the three visits. Conclusion: The significant decreasing of HA level at patients with COPD may be amarker of the effectiveness of anti-inflammatory treatment (AIT).
\end{abstract}

Keywords: COPD, Hyaluronic acid, lung.

\section{Introduction}

Chronic obstructive pulmonary disease (COPD) is a disease characterized by persistent airflow limitation that is usually progressive and is associated with an enhanced chronic inflammatory response to noxious particles or gases in the airways and the lung [1].

Chronic inflammation and airway remodeling are characteristics of COPD, abnormalities in hyaluronic acid (HA), a component of the extracellular matrix, may be involved in the pathogenesis of COPD [2].

Hyaluronic acid (HA) is a dynamic glycosaminoglycan of the lung with high daily turnover. Hyaluronidases (HYAL) degrade HA in small fragments that have pro-inflammatory properties and are implicated in lung diseases including COPD. Acute exacerbations of COPD are associated with increased levels and activity of HYAL in bronchoalveolar lavage and subsequent degradation of HA, which may contribute to airway inflammation and subsequent lung function decline during exacerbations [3].

Endogenous high molecular weight of HA plays a pivotal rolein the homeostasis of the upper and the lower airways, it is an important component of the normal airway secretions, exerts anti- inflammatory, promotes cell survival and mucociliary clearance, organizes extracellular matrix, stabilizes connective tissues, sustains healing processes and regulates tissues hydration. Hence, HA represents a promising therapeutic agent in lung diseases and other respiratory diseases [4].

The aim of this work is to study the value of hyaluronic acid (HA) in patients with chronic obstructive pulmonary disease (COPD) and follow up its level with medications in these patients.

\section{Patients and methods}

This cross-sectional study was carried out in chest department at Benha university hospitals, the samples were collected from patients in the period from Mars 2019 to December 2020.

Ethical approval was obtained by Benha University Research Ethics Review Committee and official permission obtained from head of chest department of faculty of medicine. Written informed consent was obtained from each patient of all participants.

This prospective study was being conducted in chest department at Benha university hospitals which involved two groups:

1. The main Group: 60 patients with COPD in different stages who will receive drugs or not. Then they will be subdivided into two subgroups according to the degree of bronchial obstruction into two subgroups according to (1):

- Subgroup 1 - patients who had mild COPD (I and II stage of disease) which do not require continuous use of ICS.

- $\quad$ Subgroup 2 - patients who had severe COPD (III and IV stage of disease) which requires continuous use of ICS.

2. The control Group: 20 apparently healthy individuals.

Inclusion criteria was any adult with Stable COPD patients with (FEV1/ FVC $(\% \vee \cdot>$ and apparently healthy subjects. Exclusion criteria was Exacerbation of COPD and any disease cause increase or decrease HA as (asthma, pulmonary fibrosis, hypertension, allergic rhinitis and rheumatoid arthritis

All subjects underwent history talking and clinical examination, plain chest $\mathrm{x}$-ray (postero-anterior) and (left-lateral) view and PFT (spirometery) before and after bronchodilator and it will be repeated at 3 and 6 months with the assay HA, Laboratory investigations include:CBC, ESR, Liver function tests (AST, ALT, 
and ALP. Kidney function tests (urea, creatinine), assay of the serum level of HA using ELISA and will be repeated after 3 and 6 months in COPD patients to follow up the effectiveness of medications.

\subsection{Statistical analysis}

Data were analyzed using IBM SPSS 23.0 for windows (SPSS Inc., Chicago, IL, USA) and NCSS 11 for windows (NCSS LCC., Kaysville, UT, USA). Quantitative data were expressed as mean \pm standard deviation (SD). Qualitative data were expressed as frequency and percentage. The following tests were done: Independent sample t- test of significance was used when comparing between two means. Mannwhitny test was used when comparing two means of not normally distributed data. Chi-square (X2) test of significance was used in order to compare proportions between two qualitative parameters. Fisher Exact test is a test of significance that is used in the place of chi square test in 2 by 2 tables, especially in cases of small samples. Probability (P-value): P-value $<0.05$ was considered significant, $\mathrm{P}$-value $<0.001$ was considered as highly significant and $\mathrm{P}$-value $>0.05$ was considered insignificant.

\section{Results}

The age in control group ranged from 30 to 86 years and the mean \pm SD was $59.20 \pm 15.17$ years, while in subgroup 1 the age ranged from 38 to 87 years and the mean \pm SD was $61.8 \pm 11.43$ years, and in subgroup 2 the age ranged from 30 to 90 years and the mean \pm SD was $64.30 \pm 15.63$ years. There was no statistically significant difference between the control and patients' groups as regards age

The gender was distributed in control group $13(65.0 \%)$ males, $7(35.0 \%)$ females and in subgroup 1 $19(63.3 \%)$ males,

$11(36.7 \%)$ females and in subgroup 2 18(60\%) males, $12(40 \%)$ females with no statistically significant difference

The smoking was distributed in control group $7(35.0 \%)$ non-smokers, 7(35.0\%) smokers, and $6(30 \%)$ ex-smokers and in
Subgroup (1) 14(46.7\%) non-smokers, 9(30\%) smokers, and 7(35\%) ex- smokers and in subgroup 2 $10(33.3 \%)$ non-smokers, $9(00 \%)$ smokers, and $11(36.7 \%)$ ex-smokers with no statistically significant difference

The Gold classification was distributed in subgroup (1) 14(46.7\%) Gold1, 16(53.3\%) Gold 2, and in subgroup (2).

12(40\%) Gold 3, 18(60\%) Gold 4.

The drugs usage was distributed in subgroup 1 $3(10 \%)$ LAMA only, 1(3.3\%) LABA only, 2(6.7\%) ICS only, 4(13.3\%)

ICS + LABA, and in subgroup $27(23.3 \%)$ LAMA only, 2(6.7\%) LABA only, 3(10\%) ICS only, $8(26.7 \%)$ ICS + LABA, with no statistically significant difference

The Hb, RBCs, WBCs, and ESR in control group as shown in table were statistically significant lower than patient's sub groups 1 and 2, where the two sub groups were almost equal with no statistically significant difference. There was no statistically significant difference between the controls and the two sub groups as regards Platelets, liver, and kidney function tests

There was statistically significant increase in FEV1/FVC ratio Pred. in visit two than visit one, and in visit three than visit one and two, and there was statistically significant difference between the controls and the two sub groups as regards FEV1/FVC ratio Pred., where control is the highest then subgroup 1 then subgroup 2 in the three visits.

There was statistically significant decrease in Hyaluronic acid in visit two than visit one, and in visit three than visit one and two, and there was statistically significant difference between the controls and the two sub groups as regards Hyaluronic acid, where control is the lowest then subgroup 1 then subgroup 2 in the three visits.

According to Spearman's rank correlation coefficient analysis, the Level of Hyaluronic acid was positively correlated with age, hemoglobin, red and white blood cells count in blood, and ESR and the Level of Hyaluronic acid was negatively correlated with FEV1/FVC ratio Pred.

Table ( 1 ) Comparison between the control group and patient'sSubgroups 1 and 2 as regards lab data.

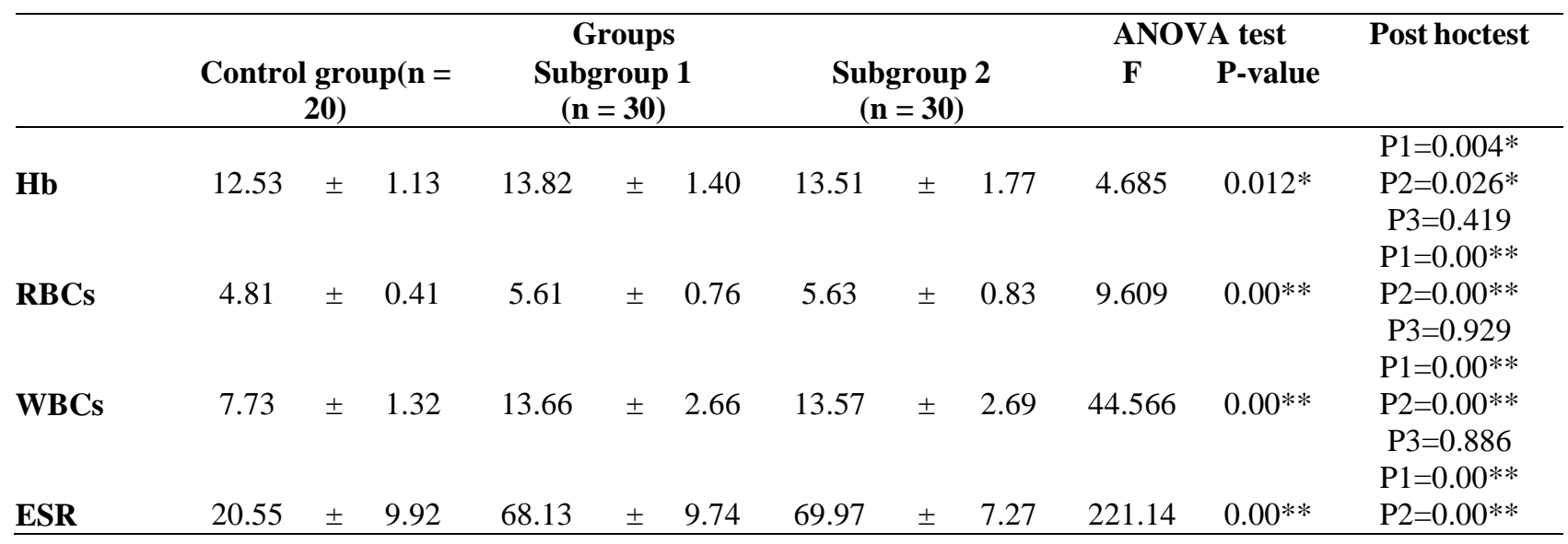




\begin{tabular}{lcccccccccccc}
\hline Platelets & 249.35 & \pm & 44.40 & 237.07 & \pm & 33.19 & 240.60 & \pm & 37.17 & 0.649 & 0.525 & P3=0.429 \\
AST & 24.90 & \pm & \pm .52 & 26.37 & \pm & 11.72 & 28.43 & \pm & 10.16 & 0.669 & 0.515 \\
ALT & 31.55 & \pm & 16.58 & 29.63 & \pm & 14.70 & 31.03 & \pm & 24.06 & 0.07 & 0.932 & \\
ALP & 4.14 & \pm & 0.52 & 4.16 & \pm & 0.47 & 4.18 & \pm & 0.38 & 0.057 & 0.945 \\
Urea & 23.95 & \pm & 9.48 & 21.27 & \pm & 6.63 & 23.87 & \pm & 8.65 & 0.971 & 0.383 & \\
Creatinine & 1.40 & \pm & 0.47 & 1.34 & \pm & 0.40 & 1.38 & \pm & 0.41 & 0.144 & 0.866 \\
\hline
\end{tabular}

P1: Control group VS Subgroup 1, P2: Control group VS Subgroup 2, P3: Subgroup 1 VS Subgroup 2

F: ANOVA Test p: $\mathrm{p}$ value for comparing between the studied groups $* \mathrm{P}$-value $<0.05$ is significant $* * \mathrm{P}$ - value $\leq 0.001$ highly significant

Table (2) Comparison between the control group and patient's Subgroups 1 and 2 as regards FEV1/FVC ratio Pred., in and between thethree visits.

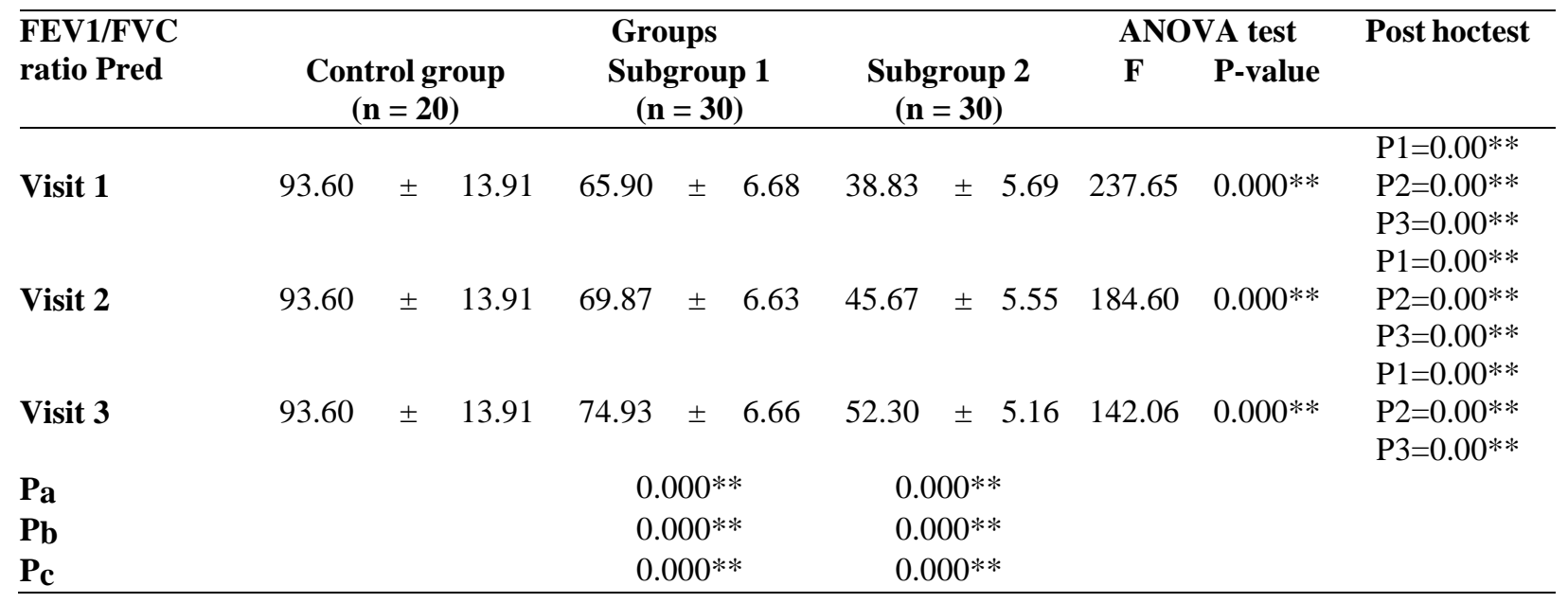

P1: Control group VS Subgroup 1, P2: Control group VS Subgroup 2, P3: Subgroup 1 VS Subgroup 2 Pa: Visit 1 VS Visit 2, Pb: Visit 1VS Visit 3, Pc: Visit 2 VS Visit 3

$* \mathrm{P}$-value $<0.05$ is significant $* * \mathrm{P}$ - value $\leq 0.001$ highly significant

Table (3) Comparison between the control group and patient'sSubgroups 1 and 2 as regards Hyaluronic acid (HA) $\mu \mathrm{g} / \mathrm{ml}$.

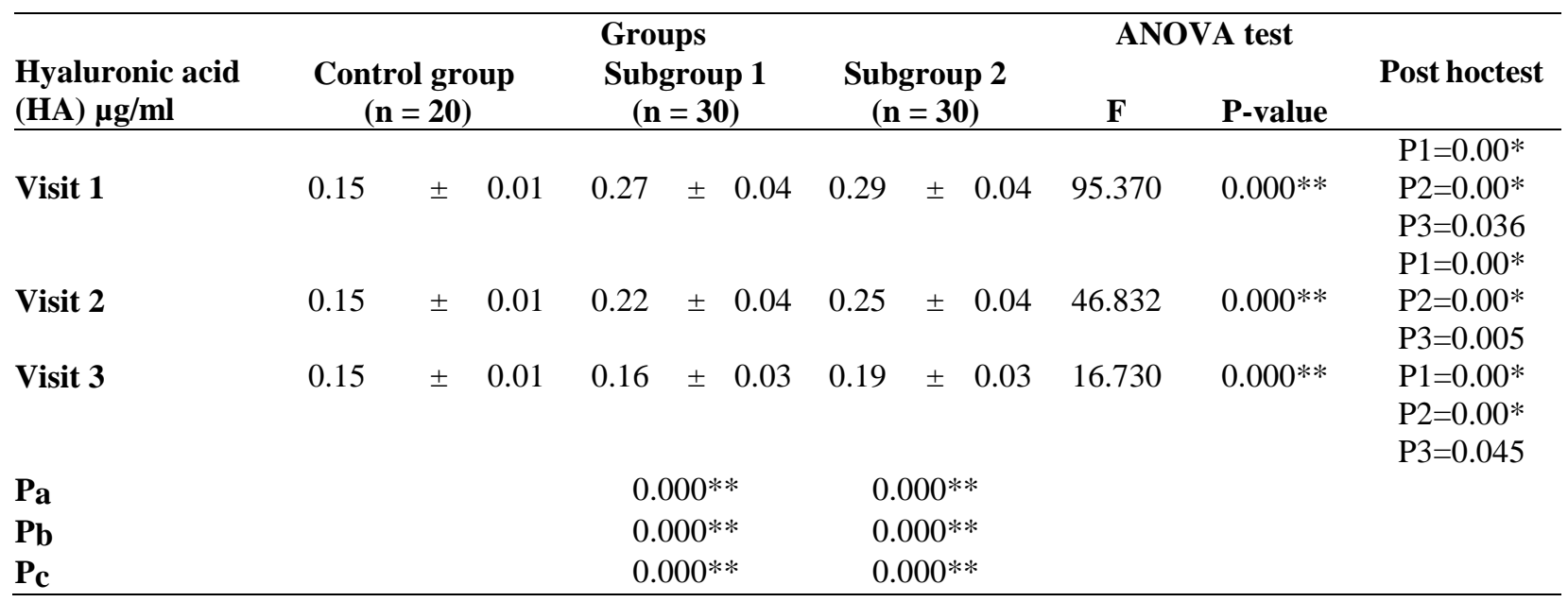

P1: Control group VS Subgroup 1, P2: Control group VS Subgroup 2, P3: Subgroup 1 VS Subgroup 2 Pa: Visit 1 VS Visit 2, Pb: Visit 1VS Visit 3, Pc: Visit 2 VS Visit 3

$* \mathrm{P}$-value $<0.05$ is significant $* * \mathrm{P}$ - value $\leq 0.001$ highly significant 
Table (4) Correlation between Levels of Hyaluronic acid with otherstudied Parameters.

\begin{tabular}{|c|c|c|}
\hline \multirow[t]{2}{*}{ Studied Parameters } & \multicolumn{2}{|c|}{ Level of Hyaluronic acid } \\
\hline & Correlation & p-value \\
\hline Age & 0.512 & $0.000 * *$ \\
\hline FEV1/FVC ratio Pred. & -0.826 & $0.000 * *$ \\
\hline $\mathbf{H b}$ & 0.245 & $0.028 *$ \\
\hline RBCs & 0.328 & $0.003^{*}$ \\
\hline WBCs & 0.934 & $0.000 * *$ \\
\hline ESR & 0.722 & $0.000 * *$ \\
\hline
\end{tabular}

$* * \mathrm{P}$ - value $\leq 0.001$ highly significant

\section{Discussion}

Our study showed highly significant between the control group and patient's Subgroups 1 and 2 as regards Hyaluronic acid (HA) $\mu \mathrm{g} / \mathrm{ml}$ level in different 3 visits of study with medication which level of hyaluronic acid decrease through these visits: subgroup $1(\mathrm{n}=30)$ visit $1=0,27 \pm 0.04 \mu \mathrm{g} / \mathrm{ml}$, visit2 $=0,22 \pm 0,04 \mu \mathrm{g} / \mathrm{ml}$, visit $3=0,16 \pm 0,03 \mu \mathrm{g} / \mathrm{ml}$ and subgroup

$2(\mathrm{n}=30)$ visit $1=0,29 \pm 0.04 \mu \mathrm{g} / \mathrm{ml}$, visit $2=0,25$ $\pm 0,04 \mu \mathrm{g} / \mathrm{ml}$, visit $3=0,19 \pm 0,03 \mu \mathrm{g} / \mathrm{ml}$. This decrease in the levels of hyaluronic acid was demonstrated in a study done by Deshpande et al. [5].

Another study was that HA serum levels could be modified by treatment, since it has been shown that HA turnover is altered by glucocorticoids and LABAs, and the majority of COPD patients that participated in the present study were under treatment with ICSs ( $81 \%$ of the patients) or with ICS+LABA combination $(61.3 \%$ of the patients).it was demonstrated in a study done by Karakiulakis and Michael[6].

In contrast, Papakonstantinou et al. [7]. reported the results of a study of the analysis of HA according to treatment, even if the groups that were receiving treatment and the groups that were not receiving treatment were not balanced for numbers of patients, we could not observe any significant differences in HA levels between the groups receiving ICSs, systemic corticosteroids or ICS +LABA and the groups that were not receiving any treatment.

In the present study also, we provide evidence that serum levels of HA are significantly increased at moderate and severe exacerbations of COPD compared with the stable state of the disease. HA levels at exacerbation were not related to bacterial infections or to markers of acute inflammation such as leukocyte counts and C-reactive protein, indicating that COPD exacerbations are associated with increased degradation of HA leading to the formation of short HA fragments. In the last two decades extensive research has shown that signaling by short HA fragments contributes to the accumulation of immune cells in inflammatory sites and the subsequent release of pro-inflammatory cytokines and metalloelastases that was demonstrated in a study done by Stolz et al. [8].
In contrast, Pertseva.T.O et al. [9]. Correlations between the HA and the severity of the disease has not been determined $(\mathrm{p}=0.355)$, while direct correlation was determined between the level of HA and FEV1.

Also, we show that serum levels of HA were significantly associated with pro-collagen types III and VI, the major collagens found in the lung interstitium and in large bronchi. As well as with the degradation products of collagen type IV and collagen type VI that is mainly found in the basement membranes. These results indicate that $\mathrm{HA}$, in line with collagens, is a part of the increased ECM turnover in COPD, a process that determines disease severity and clinically relevant outcomes that was demonstrated in a study done by Garantziotis et al. [10].

Here, we provide evidence, that HYAL activity in the serum of 60 COPD patients was negatively correlated with FEV1\% predicted and with survival time. These results indicate that enzymatic degradation of physiologically occurring high-molecular weight HA in COPD patients is associated with airflow limitation and impairment of lung function that was demonstrated in a study done by Papakonstantinou et al. [7].

Regarding markers of inflammation, HA serum levels were significantly correlated with white blood cells count in blood, ESR and C - reactive protein $(p<0.001)$ in control group as shown in table were statistically significant lower than patients' sub groups 1 and 2, where the two sub groups were almost equal with no statistically significant difference. There was no statistically significant difference between the controls and the two sub groups as regards Platelets, liver, and kidney function tests. There was no significant correlation of serum levels of HA with smoking history that was demonstrated in a study done by Stolz et al. [8].

Our study has a few limitations. Measurements of HA serum concentration by ELISA do not provide information about the size of HA. Unfortunately, to date, there is no appropriate methodology to estimate the size of such a large glycosaminoglycan in serum samples. Thus, parallel measurements of HYAL-1, which is the main HYAL detected in human serum, provide the best indication for the size of HA.

A major strength relates to the two-stage design with two well-matched cohorts. The discovery cohort 
was designed to maximize the chances to establish HA as a potential molecule associated with disease severity and outcome, and to minimize unnecessary waste of patient samples. Additional strengths are the longitudinal sampling undertaken at two points in time (at stable state, and at follow-up) and the longitudinal clinical data that enabled us to determine disease outcomes.

\section{Conclusion}

Determination of levels of such marker of systemic like HA can be used as parameters for further evaluation of the clinical stability of patients during their long-term follow up. The significant decreasing of HA level at patients with COPD may be a marker of the effectiveness of anti-inflammatory treatment (AIT).

\section{References}

[1] GOLD. Global Strategy for the Diagnosis, Management and Prevention of COPD.vol.8,pp.8-19,2020

[2] JH.Vernooy and S.Hendriks. Enhanced levels of hyaluronan in lungs of patients with COPD: Relationship with lung function and local inflammation. Thorax.vol.60(2),pp.114-19 ,2005.

[3] E.Papakonstantinou, M.Roth and I.Klagas. COPD exacerbations are associated with proinflammatory degradation of hyaluronic acid. Chest.vol.148,pp.1497-1507,2015.

[4] G.M.Turino, S.Ma, Y.Y.Lin and J.O.Cantor. The therapeutic potential of hyaluronan in COPD.vol. 153(4),pp.792-98,2018.
[5] M.Deshpande, S.Papp and L.Schaffer. (Hydrocortisone and triiodothyronine regulate hyaluronate synthesis in a tissue-engineered human dermal equivalent through independent pathways. J Biosci Bioeng.vol.119,pp. 226-236, 2015.

[6] G.Karakiulakis and Michael Roth. Muscarinic Receptors and Their Antagonists in COPD: Anti-Inflammatory and Antiremodeling Effects", Mediators of Inflammation.vol.8,pp, 409580,2012.

[7] E.Papakonstantinou , D.Schumann, D.Leeming , M.Tamm , M.Karsdal and D.Stolz.. Extracellular matrix molecules are differentially associated with airflow obstruction and lung parenchymal destruction in COPD. Eur.Resp.J.vol. 54(63),pp.167781,2019 .

[8] D.Stolz, DJ.Leeming and JH.Kristensen. Systemic biomarkers of collagen and elastin turnover are associated with clinically relevant outcomes in COPD. Chest.vol. 151,pp. 47-59. ,2017.

[9] T.O.Pertseva, L.I.Konopkina and B.O.Basina. Diagnostic and Prognostic value of Hyaluronic acid in patients with COPD. The Pharma Innovation Journal.vol.4(2),pp.25-27,2015.

[10] S.Garantziotis, M.Brezina, P.Castelnovo, and L.Drago. The role of hyaluronan in the pathobiology and treatment of respiratory disease. Am J Physiol Lung Cell Mol Physiol.vol. (310),pp.785-95,2016. 